\title{
Cryo-electron tomography of the mammalian synapse
}

Running title: Cryo-electron tomography of synaptosome preparations

Rubén Fernández-Busnadiego

Department of Molecular Structural Biology, Max Planck Institute of Biochemistry

Am Klopferspitz 18, 82152 Martinsried, Germany

Correspondence to: $\underline{\text { ruben@biochem.mpg.de }}$

\section{Summary}

Characterizing the detailed structure of the mammalian synapse is of crucial importance to understand its mechanisms of function. Here I describe a protocol to study synaptic architecture by cryo-electron tomography (cryo-ET), a powerful electron microscopy technique that enables 3D visualization of unstained, fully hydrated cellular structures at molecular resolution. The protocol focuses on purified synaptic terminals ("synaptosomes"), currently the most suitable preparation to analyze mammalian synaptic architecture by cryo-ET.

\section{Keywords}

Cryo-electron microscopy, cryo-EM, neuron, tissue fractionation, synaptic vesicle, postsynaptic density

\section{Introduction}

Cryo-ET is the technique of choice to study the molecular architecture of the cell at nanometer resolution. To that end the samples need to be vitrified, i.e. frozen at fast cooling rates that prevent the reorganization of water molecules into crystals, and instead form amorphous ice. Vitrified samples are mounted on a cryo-electron microscope capable of keeping them close to liquid 
nitrogen temperature during imaging. Because no contrasting agents such as heavy metals are applied, the (phase) contrast of these images arises directly from the biological densities. Images of the object of interest are recorded from different angles by tilting the specimen stage, and these images are then computationally recombined into a 3D tomogram. Thus, this technique allows the $3 \mathrm{D}$ visualization of fully hydrated, unstained cells in which the fine structural detail is preserved to a much greater extent than with classical EM techniques (Asano et al., 2015a).

However, cryo-ET also poses additional challenges in terms of sample preparation. Because the specimens are embedded in vitreous ice, they are much more difficult to section by mechanical means than their plastic-embedded counterparts. Vitreous sectioning is nevertheless possible and has been successfully applied to the study of mammalian synapses in organotypic slices (FernandezBusnadiego et al., 2010; Zuber et al., 2005), but the yield of this technique is low and the vitreous sections suffer from artifacts such as substantial compression along the cutting direction. Dissociated primary neuronal cultures are also amenable for cryo-ET, as they can be grown on EM grids and neurites can be imaged directly without the need of thinning procedures (Asano et al., 2015b; Lucic et al., 2007). However, it is hard to find synapses in these preparations as they may preferentially form in areas too thick for direct imaging. It is also possible to study the molecular organization of certain synaptic components using isolated synaptic membranes (Zuber and Unwin, 2013).

However, nowadays synaptosomes are arguably the experimental system offering the best compromise between synapse yield and structural preservation for cryo-ET (Fernandez-Busnadiego et al., 2011). Synaptosomes consist on portions of the pre- and postsynaptic terminals that reseal upon nervous tissue homogenization and are held together by the molecules of the synaptic cleft. These isolated synapses remain largely functional, as they maintain mitochondrial respiration and can be stimulated for $\mathrm{Ca}^{2+}$-dependent neurotransmitter release upon membrane depolarization (Whittaker, 1993). One should also be aware of the limitations of this system, as synaptosomes 
cannot carry out sustained neurotransmitter release and delicate structural features such as the actin cytoskeleton may be altered during preparation. Nevertheless, our studies showed that synaptic architecture in synaptosomes was not only structurally comparable to that of tissue slices (Fernandez-Busnadiego et al., 2010), but also a proxy for the electrophysiological properties of the brain (Fernandez-Busnadiego et al., 2013). In the following I present a detailed protocol on the extraction and vitrification of murine synaptosomes for cryo-ET studies.

\section{Materials}

\subsection{Synaptosome extraction}

For all buffers use ultrapure water and analytical grade reagents. The amounts given here are for one mouse brain, which provides many times more material than needed in a typical cryo-ET experiment. The protocol is based on the Percoll gradient method (Dunkley et al., 1988; Dunkley et al., 2008; Godino Mdel et al., 2007).

1. Sucrose buffer (5x): 5 mM EDTA, 1.25mM DL-DTT, 20 mM HEPES, 1.6 M sucrose. Prepare the following solutions separately: $0.25 \mathrm{M}$ EDTA (dissolve $2.33 \mathrm{~g}$ in $25 \mathrm{ml}$ of water; add $\mathrm{NaOH}$ pellets to allow dissolution and adjust $\mathrm{pH}$ to 7.4 ) and $0.1 \mathrm{M} \mathrm{DL-DTT}$ (dissolve $0.15 \mathrm{~g}$ in $10 \mathrm{ml}$ of water). Dissolve $2.38 \mathrm{~g}$ of HEPES in $400 \mathrm{ml}$, adjust $\mathrm{pH}$ to 7.4 , and then add $273.84 \mathrm{~g}$ of sucrose (stir for $\sim 1 \mathrm{~h}$ at $30^{\circ} \mathrm{C}$ to allow dissolution). Add $10 \mathrm{ml}$ of $0.25 \mathrm{M}$ EDTA and $6.25 \mathrm{ml}$ of $0.1 \mathrm{M}$ DL-DTT and adjust the volume to $500 \mathrm{ml}$ with water. This solution can be stored at $-80^{\circ} \mathrm{C}$. Make $50 \mathrm{ml}$ aliquots and freeze.

2. Homogenization buffer: Dissolve 5 cOmplete mini EDTA-free protease inhibitor tablets (Roche) in $7 \mathrm{ml}$ of water. Immediately before use add $63 \mathrm{ml}$ of 1 x sucrose buffer.

3. Percoll solutions: For $3 \% / 10 \% / 23 \%$ Percoll solutions mix $10 \mathrm{ml} 5 \mathrm{x}$ sucrose buffer respectively with 1.5/5/11.5 $\mathrm{ml}$ Percoll and adjust the volume to $40 \mathrm{ml}$ with water. Adjust $\mathrm{pH}$ to 7.4 at $4{ }^{\circ} \mathrm{C}$ and bring to $50 \mathrm{ml}$ with water. Work with Percoll in sterile conditions. These solutions can be stored for a week at $4{ }^{\circ} \mathrm{C}$. 
4. Percoll gradients: Lay $2 \mathrm{ml}$ of $3 \%$ Percoll solution with a pipette. Wash peristaltic pump with $10 \%$ Percoll solution and load $2 \mathrm{ml}$ into the tube by pressing a glass pipette coupled to the pump against the bottom of the tube. Repeat this step for the $23 \%$ Percoll solution. Make two gradients.

5. Saline buffer: $140 \mathrm{mM} \mathrm{NaCl}, 5 \mathrm{mM} \mathrm{KCl}, 5 \mathrm{mM} \mathrm{NaHCO} 3,1.2 \mathrm{mM} \mathrm{NaH}_{2} \mathrm{PO}_{4}, 1 \mathrm{mM} \mathrm{MgCl}, 10 \mathrm{mM}$ glucose, $10 \mathrm{mM}$ HEPES. For $500 \mathrm{ml}$, add the following amounts to $400 \mathrm{ml}$ of water: $4.1 \mathrm{~g} \mathrm{NaCl}, 0.19 \mathrm{~g}$ $\mathrm{KCl}, 0.21 \mathrm{~g} \mathrm{NaHCO}_{3}, 0.08 \mathrm{~g} \mathrm{NaH}_{2} \mathrm{PO}_{4}-\mathrm{H}_{2} \mathrm{O}, 0.10 \mathrm{~g} \mathrm{MgCl}_{2}-6 \mathrm{H}_{2} \mathrm{O}, 0.9 \mathrm{~g}$ glucose, $1.19 \mathrm{~g}$ HEPES. Adjust pH to 7.4 and bring to $500 \mathrm{ml}$ with water. This solution can be stored for a week at $4{ }^{\circ} \mathrm{C}$.

6. Murine dissection surgical kit

7. Teflon-glass homogenizer

\subsection{Synaptosome vitrification}

1. Copper EM grids coated with a holey carbon support

2. $10 \mathrm{~nm}$ BSA-coated gold fiducial markers (Aurion)

3. Whatman \#1 filter paper

\section{Methods}

\subsection{Synaptosome extraction}

It is critical to perform this procedure at $4{ }^{\circ} \mathrm{C}$. Always work on ice using pre-cooled tools, tubes and solutions.

1. Remove the brain from the skull and rinse in homogenization buffer.

2. Remove the cerebellum. Separate the brain hemispheres and carefully remove the white matter and blood vessels.

3. Homogenize in $4 \mathrm{ml}$ of homogenization buffer (see Note 1 and Note 2). 
4. Load the homogenate in a $50 \mathrm{ml}$ tube and centrifuge $2 \mathrm{~min}$ at $3000 \mathrm{~g}$ ( $5000 \mathrm{rpm}$ on a SS-34 rotor). Pour the supernatant (S1) into a new centrifuge tube making sure that no pellet (P1) is transferred.

5. Gently rock the tube alternatively clock and anticlockwise to detach P1 from the tube wall and resuspend carefully using a plastic pipette (see Note 3). Balance with homogenization buffer and centrifuge again $2 \mathrm{~min}$ at $3000 \mathrm{~g}$ (5000 rpm on a SS-34 rotor).

6. Pour supernatant $\left(\mathrm{S}^{\prime}\right)$ into a new centrifuge tube making sure that no pellet ( $\left.\mathrm{P} 1^{\prime}\right)$ is transferred. Combine S1 and S1' and centrifuge $15 \mathrm{~min}$ at $9200 \mathrm{~g}$ (8800 rpm on a SS-34 rotor).

7. Remove the supernatant (S2) by aspiration and carefully resuspend the pellet (P2) in $3 \mathrm{ml}$ sucrose buffer.

8. Load the Percoll gradients with resuspended P2 very slowly to avoid disturbing the gradients. Centrifuge $12 \mathrm{~min}$ at $18700 \mathrm{~g}$ (12500 rpm on a SS-34 rotor).

9. Slowly remove the synaptosome band at the $10-23 \%$ Percoll interface using a glass pipette and place in a new centrifuge tube.

10. Fill up each tube with $30 \mathrm{ml}$ saline solution and centrifuge $12 \mathrm{~min}$ at $18700 \mathrm{~g}$ (12500 rpm on a SS34 rotor) to wash the Percoll away.

11. Remove the supernatant (S3) very carefully and resuspend the pellet (P3) in as little saline solution as possible.

12. Determine protein concentration and dilute in saline solution as needed to obtain $1 \mathrm{mg} / \mathrm{ml}$. Keep at $4{ }^{\circ} \mathrm{C}$.

\subsection{Synaptosome vitrification by plunge freezing}

Given their relatively small size (typically a few hundred nanometers in diameter), synaptosomes can be successfully vitrified by plunge freezing into a fast cooling cryogen such as liquid ethane cooled down to liquid nitrogen temperature. 
1. It is beneficial to mix gold fiducial particles with synaptosomes to aid the computational alignment of tomographic projections (Kremer et al., 1996). BSA-coated gold fiducials minimize aggregation and interactions with the sample. The fiducials need to be centrifuged prior to mixing with synaptosomes to (i) remove the sodium azide used as preservative, and (ii) achieve the desired final concentration. Spin $125 \mu$ l of $10 \mathrm{~nm}$ BSA-coated gold fiducial markers for $90 \mathrm{~min}$ at $13000 \mathrm{rpm}$ using a tabletop centrifuge. Carefully remove $\sim 100 \mu \mathrm{l}$ of supernatant without disturbing the pellet so that the remaining volume is $\sim 25 \mu \mathrm{l}$ ( $5 \mathrm{x}$ concentrated gold particles). Resuspend with another $25 \mu \mathrm{l}$ of saline buffer and incubate at $37^{\circ} \mathrm{C}$.

2. Glow discharge the EM grids using a plasma cleaner to render them hydrophilic.

3. Incubate synaptosomes at $37^{\circ} \mathrm{C}$ in a water bath for $30 \mathrm{~min}$ prior to vitrification so that they regain their physiological properties. During this incubation it is also possible to apply pharmacological treatments of interest.

4. Mix $50 \mu$ l of gold fiducials resuspended in saline buffer with $50 \mu$ of synaptosomes.

5. Mount a glow-discharged EM grid in the vitrification device and apply $3 \mu \mathrm{l}$ of the synaptosome/gold fiducial mixture on the side of the grid coated by the carbon film. Incubate $10 \mathrm{~s}$ to allow synaptosomes to settle on the carbon film of the EM grid.

6. Blot the excess buffer from the grid using Whatman \#1 filter paper. If the vitrification device allows single-sided blotting, blot only from the back of the grid (the opposite side where the sample was deposited). Plunge immediately into the cryogen, e.g. liquid ethane or 1:2 ethane-propane mixture (see Note 4).

\subsection{Cryo-ET imaging of synaptosomes}

Synaptosomes can be imaged by cryo-ET using any cryo-electron microscope equipped with a tilting stage and automated tomography software such as SerialEM (Mastronarde, 2005). For best results, 
use a $300 \mathrm{kV}$ microscope equipped with a field emission gun, energy filter and a direct electron detector.

1. Load the EM grid containing vitrified synaptosomes in the cryo-electron microscope. Using low magnification ( 125x) identify regions of suitable ice thickness (see Note 5).

2. The most abundant feature in this preparation are isolated presynaptic terminals without an associated postsynaptic counterpart (Figure 1A, B, black arrowheads), which may not be suitable for studies on synaptic transmission (see Note 6). Cellular debris and isolated mitochondria can also be seen. Figure $\mathbf{1 C}$ and $\mathbf{D}$ show a typical synaptosome consisting on a presynaptic terminal associated to a much smaller postsynaptic compartment. Presynaptic terminals in synaptosomes often contain hundreds of synaptic vesicles, some of which may be clathrin-coated (Figure 1E), mitochondria and ER membranes.

3. For optimal tomogram quality, synaptosomes should be embedded in a layer of ice as thin as possible, surrounded by sufficient fiducial markers (see Note 7), placed within the holes of the carbon film and in areas allowing a tilt range of at least $\pm 60^{\circ}$.

\section{Notes}

Note 1: Homogenization must be careful to minimize heating. For the first stroke, the homogenizer should be started at minimum speed once the piston is inside the solution. After this stroke the speed should be increased slowly to $900 \mathrm{rpm}$ and homogenization should proceed until no small parts of tissue are visible (7 strokes maximum). When homogenization is completed, reduce the speed to minimum and remove the piston from the solution.

Note 2: Rapid dissection and homogenization are crucial for the purification of functional synaptosomes. If more than one brain is being used, the procedure can be paused at this point and repeated for subsequent brains. The homogenates can then be pooled. 
Note 3: Gentle resuspension of the pellets is mandatory to preserve synaptosome integrity. The pellet can be detached from the tube by softly rocking it alternatively clock and anticlockwise. Take up the pellet with a pipette and flush it out, making sure that the pipette tip makes tight contact with the bottom of the tube to maximize the efficiency of the resuspension and minimize the number of iterations (the process should be repeated until no visible pieces are visible, ideally not more than three times). If the pellet to be resuspended is larger than one pipette volume, use fresh tubes for resuspension to avoid disturbing already resuspended material.

Note 4: whereas pure ethane solidifies at liquid nitrogen temperature, a 1:2 ethane-propane mixture remains liquid and still allows good vitrification (Tivol et al., 2008). However, this mixture can also be liquid above the vitrification temperature, so it must be cooled continuously by liquid nitrogen to ensure proper freezing.

Note 5: synaptosomes tend to form large clumps that are too thick for direct cryo-ET imaging. Appropriate resuspension prior to freezing can alleviate this problem.

Note 6: to speed up the search of synaptosomes suitable for cryo-ET imaging, correlative cryo-light microscopy (Fukuda et al., 2014; Schellenberger et al., 2014; Zhang, 2013) can be applied provided that synaptosomes have been loaded with fluorescent dyes (e.g. FM1-43) or were extracted from animals expressing fluorescently labeled synaptic proteins.

Note 7: fiducial gold markers allow optimal alignment of tomographic projections, but current feature-based alignment methods such as those implemented in IMOD (Kremer et al., 1996) also provide good results.

\section{Acknowledgments}

I wish to thank Eri Sakata for the critical reading of this manuscript. R. F.-B. is supported by the FP7 GA ERC-2012-SyG_318987-ToPAG grant from the European Commission. 
Figure

Figure 1
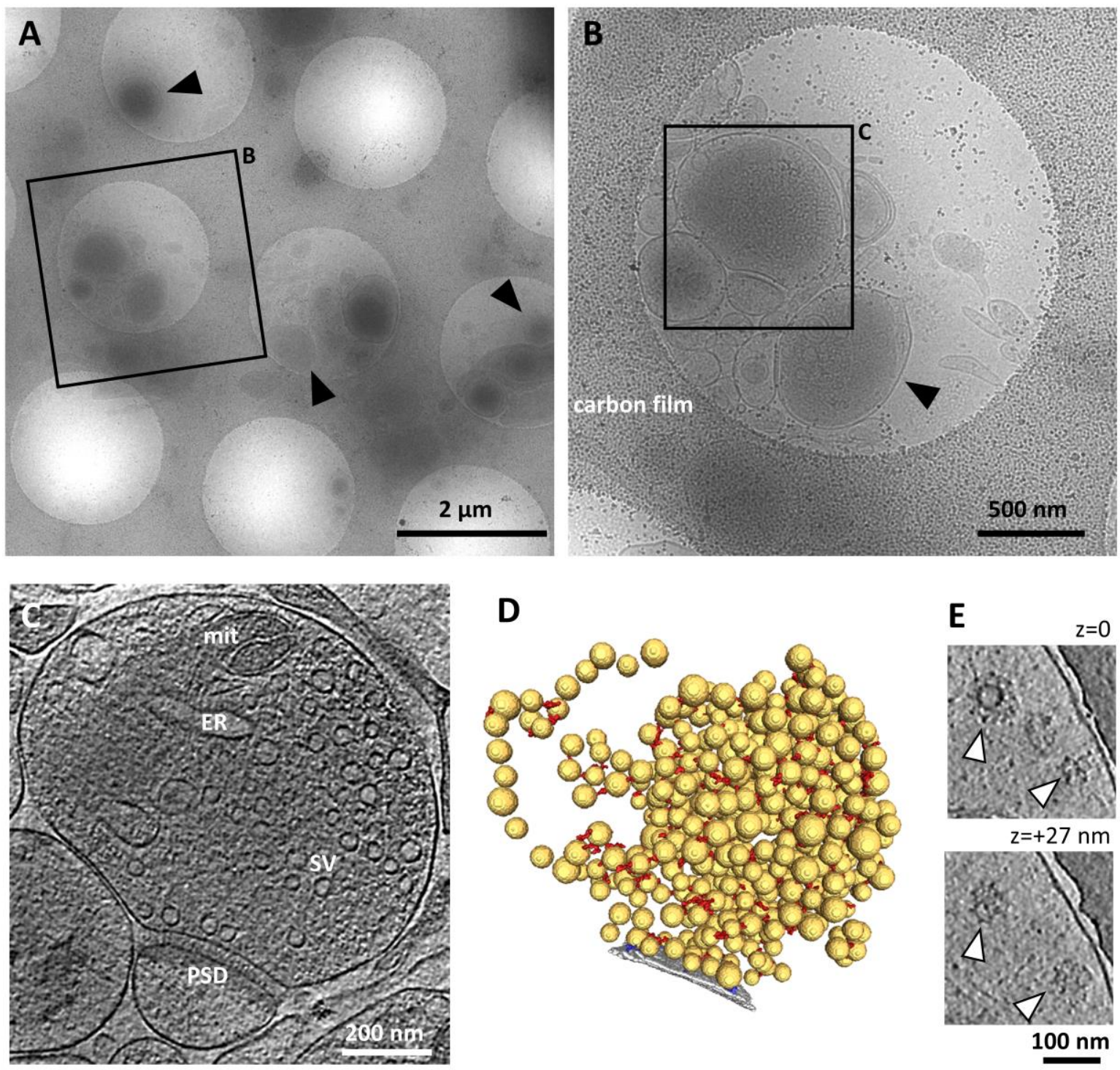
Figure 1: cryo-ET imaging of rat synaptosomes. A, B) 2D images of increasing magnification of an EM grid coated with a holey carbon film containing vitrified frozen-hydrated rat synaptosomes. Black arrowheads mark isolated presynaptic terminals without a postsynaptic counterpart. C) Tomographic slice of the synaptosome marked in B. The presynaptic terminal contains synaptic vesicles (SV), a mitochondrion (mit) and ER membranes (ER). On the postsynaptic terminal the postsynaptic density (PSD) is visible. D) Semi-automated 3D rendering of the area within $250 \mathrm{~nm}$ of the active zone of the synaptosome in C, showing synaptic vesicles (yellow), the active zone (grey) and the fine filaments linking synaptic vesicles to each other (red) and to the active zone (blue). E) Tomographic slices of another synaptosome depicting the same region in $\mathrm{x}$ and $\mathrm{y}$ at different $\mathrm{z}$ heights ( $\mathrm{z}=0 \mathrm{~nm}$ and $\mathrm{z}=+27 \mathrm{~nm}$ respectively). White arrowheads mark a clathrin-coated synaptic vesicle (top) and an empty clathrin coat (bottom). Tomographic slices are $2.7 \mathrm{~nm}$-thick (modified from Ref. Fernandez-Busnadiego et al., 2010 with permission of Rockefeller University Press). 


\section{References}

Asano, S., Engel, B.D., and Baumeister, W. (2015a). In Situ Cryo-Electron Tomography: A PostReductionist Approach to Structural Biology. J Mol Biol.

Asano, S., Fukuda, Y., Beck, F., Aufderheide, A., Forster, F., Danev, R., and Baumeister, W. (2015b).

Proteasomes. A molecular census of 265 proteasomes in intact neurons. Science $347,439-442$.

Dunkley, P.R., Heath, J.W., Harrison, S.M., Jarvie, P.E., Glenfield, P.J., and Rostas, J.A. (1988). A rapid

Percoll gradient procedure for isolation of synaptosomes directly from an S1 fraction: homogeneity and morphology of subcellular fractions. Brain Res 441, 59-71.

Dunkley, P.R., Jarvie, P.E., and Robinson, P.J. (2008). A rapid Percoll gradient procedure for preparation of synaptosomes. Nat Protoc 3, 1718-1728.

Fernandez-Busnadiego, R., Asano, S., Oprisoreanu, A.M., Sakata, E., Doengi, M., Kochovski, Z., Zurner, M., Stein, V., Schoch, S., Baumeister, W., et al. (2013). Cryo-electron tomography reveals a critical role of RIM1alpha in synaptic vesicle tethering. J Cell Biol 201, 725-740.

Fernandez-Busnadiego, R., Schrod, N., Kochovski, Z., Asano, S., Vanhecke, D., Baumeister, W., and Lucic, V. (2011). Insights into the molecular organization of the neuron by cryo-electron tomography. J Electron Microsc (Tokyo) 60 Suppl 1, S137-148.

Fernandez-Busnadiego, R., Zuber, B., Maurer, U.E., Cyrklaff, M., Baumeister, W., and Lucic, V. (2010). Quantitative analysis of the native presynaptic cytomatrix by cryoelectron tomography. J Cell Biol $188,145-156$.

Fukuda, Y., Schrod, N., Schaffer, M., Feng, L.R., Baumeister, W., and Lucic, V. (2014). Coordinate transformation based cryo-correlative methods for electron tomography and focused ion beam milling. Ultramicroscopy 143, 15-23.

Godino Mdel, C., Torres, M., and Sanchez-Prieto, J. (2007). CB1 receptors diminish both $\mathrm{Ca}(2+)$ influx and glutamate release through two different mechanisms active in distinct populations of cerebrocortical nerve terminals. J Neurochem 101, 1471-1482. 
Kremer, J.R., Mastronarde, D.N., and McIntosh, J.R. (1996). Computer visualization of threedimensional image data using IMOD. J Struct Biol 116, 71-76.

Lucic, V., Kossel, A.H., Yang, T., Bonhoeffer, T., Baumeister, W., and Sartori, A. (2007). Multiscale imaging of neurons grown in culture: from light microscopy to cryo-electron tomography. J Struct Biol 160, 146-156.

Mastronarde, D.N. (2005). Automated electron microscope tomography using robust prediction of specimen movements. J Struct Biol 152, 36-51.

Schellenberger, P., Kaufmann, R., Siebert, C.A., Hagen, C., Wodrich, H., and Grunewald, K. (2014). High-precision correlative fluorescence and electron cryo microscopy using two independent alignment markers. Ultramicroscopy 143, 41-51.

Tivol, W.F., Briegel, A., and Jensen, G.J. (2008). An improved cryogen for plunge freezing. Microsc Microanal 14, 375-379.

Whittaker, V.P. (1993). Thirty years of synaptosome research. Journal of neurocytology 22, 735-742. Zhang, P. (2013). Correlative cryo-electron tomography and optical microscopy of cells. Curr Opin Struct Biol 23, 763-770.

Zuber, B., Nikonenko, I., Klauser, P., Muller, D., and Dubochet, J. (2005). The mammalian central nervous synaptic cleft contains a high density of periodically organized complexes. Proc Natl Acad Sci U S A 102, 19192-19197.

Zuber, B., and Unwin, N. (2013). Structure and superorganization of acetylcholine receptor-rapsyn complexes. Proc Natl Acad Sci U S A 110, 10622-10627. 